\title{
LOCAL HOLONOMY GROUPS OF INDUCED CONNECTIONS ${ }^{1}$
}

\author{
MU-CHOU LIU
}

\begin{abstract}
There are two naturally induced connections on the tangent bundle, the so called Jacobi connection and the Sasaki connection. By using the elementary theory of systems of linear differential equations, we completely determine the local holonomy group of these two induced connections, and find some relation to the local holonomy group of the manifold itself. There is an induced cornection on the vector bundle of linear maps of the fibers. We also investigate the properties of the holonomy group of this bundle.
\end{abstract}

1. Introduction. Suppose $M$ is a Riemannian manifold with $\operatorname{dim} M=m$. There exist two naturally induced metrics on the tangent bundle $\tau: T M \rightarrow$ $M$, the Sasaki metric [5] and the induced pseudo-Riemannian metric [6], [8]. In [8] Yano and Kobayashi determined the holonomy group $\Phi(T \nabla)$. In this paper, we shall determine the local holonomy group $\Phi^{*}(u, T M)$ of the pseudo-Riemannian connection by using a direct argument, based on the elementary uniqueness and existence theorems of a system of linear differential equations. By introducing an orthonormal base for the vector fields on $M$, we shall be able to express components $\left\{\Gamma_{J K}^{I}\right\}$ of the Sasaki connection in a much simpler form. It will enable us to find a simple expression for any element of the local holonomy group of the Sasaki connection. Suppose $\Pi: E \rightarrow M$ and $\rho: F \rightarrow M$ are vector bundles over $M$. From [2], there exists an induced connection on $L(\Pi ; \rho): L(E, F) \rightarrow M$, the vector bundle of linear maps of the fibers. Suppose $\Phi(\Pi)$ and $\Phi(\rho)$ are holonomy groups of $\Pi$ and $\rho$ respectively. We shall show that the holonomy group $\Phi(L(\Pi, \rho))$ of $L(\Pi, \rho)$ is a subgroup of $\Phi(\Pi) \otimes \Phi(\rho)$, and then apply this result to existence theorems on infinitesimal isometry, affine fiber map and parallel vector field on $M$.

2. $T M$ admits pseudo-Riemannian metric. Let $U$ be a connected open set of a manifold $M$ with connection $\Gamma$, and let $\Gamma_{U}$ be the induced

Received by the editors June 27, 1972 and, in revised form, February 21, 1973.

AMS (MOS) subject classifications (1970). Primary 53B05, 55F10.

Key words and phrases. Pseudo-Riemannian connection, Sasaki connection, holonomy group, parallel vector field, parallel section, affine fiber map, parallel translation, vector bundle, tangent bundle.

${ }^{1}$ Work partially supported by NSF Grant GP 12760.

(c) American Mathematical Society 1974 
connection on $U$. If $\Phi^{0}(p, U)$ is the restricted holonomy group with reference point $p$ in $U$, then the local holonomy group $\Phi^{*}(p, M)$ with reference point $p$ is defined to be the intersection $\bigcap \Phi^{0}(p, U)$ where $U$ runs through all connected neighborhoods of $p$. If $\left\{U_{k}\right\}$ is a sequence of connected neighborhoods of $p$ such that $U_{k} \supset \bar{U}_{k+1}$ and $\bigcap_{k=1}^{\infty} U_{k}=\{p\}$. Then we have $\Phi^{*}(p, M)=\Phi^{0}\left(p, U_{k}\right)$ for sufficiently large $k$. Suppose now that $M$ is a Riemannian manifold, and that the tangent bundle $\tau: T M \rightarrow M$ admits the pseudo-Riemannian metric. Then there exist simply connected neighborhoods $U$ in $M$ and $V$ in $T M$ such that (i) $U$ is a normal neighborhood of $p$ in $M$ with coordinates $\left\{x^{i}\right\}$, (ii) $\tau(V)=U$, (iii) $\Phi^{*}(p, M)=\Phi^{0}(p, U)$, and (iv) $\Phi^{*}(u, T M)=\Phi^{0}(u, V)$ where $u \in \tau^{-1}(p)$.

If $\left\{\Gamma_{j k}^{i}\right\}$ denote the components of the Riemannian connection, then $\Gamma_{j k}^{i}(p)=0$ for all $i, j, k$. If $Z=\left\{Z^{i}\right\}$ is a tangent vector, $\Gamma(Z)$ denotes the $m \times m$ matrix $\left(\sum_{j=1}^{m} \Gamma_{j k}^{i} Z^{j}\right)$. Let $C(p, U)$ denote the loop space at $p$ contained in $U$.

LEMMA. $\Phi^{*}(p, M)=\left\{\exp \int_{0}^{1} \Gamma(d c / d t) d t \mid c \in C(p, U)\right\}$.

ProOF. If $Y(t)=\left\{Y^{i}(t)\right\}$ is a parallel vector field along $c(t)=\left\{c^{i}(t)\right\}$, then $Y^{i}(t)$ must satisfy the following equations

$$
\frac{d Y^{i}}{d t}+\sum_{j, k=1}^{m} \Gamma_{j k}^{i} \frac{d c^{j}}{d t} Y^{k}=0 \text { for } i=1,2, \cdots, m .
$$

The solution of this system of equations is given by

$$
Y(t)=\left\{\exp \int_{0}^{t} \Gamma\left(\frac{d c}{d s}\right) d s\right\} Y(0) .
$$

If the curve $c(t)$ defines an element $a \in \Phi^{*}(p, M)$, then it follows immediately that $a=\exp \int_{0}^{1} \Gamma(d c / d t) d t$. Q.E.D.

Let $\alpha(t)=\left\{c^{i}(t), \xi^{i}(t)\right\}$ be a curve in $V$, and let $c=\tau(\alpha)$ be a curve in $U$. Suppose that $\alpha(t)$ defines an element $\tilde{a} \in \Phi^{*}(u, T M)$, and that $c(t)$ defines an element $a \in \Phi^{*}(p, M)$. Let $b$ be an element of $\operatorname{gl}(m, R)$ defined by

$$
b=A(1) \int_{0}^{1} A(t)^{-1} R\left(\frac{d c}{d t}, \xi\right) A(t) d t,
$$

where $A(t)=\int_{0}^{t}-\Gamma(d c / d s) d s$.

THEOREM 1. Every element $\tilde{a}$ of the holonomy group $\Phi *(u, T M)$ is given in the form $\tilde{a}=\left(\begin{array}{cc}a & 0 \\ b & a\end{array}\right)$ where $a \in \Phi^{*}(p, M)$ and $b \in \operatorname{gl}(m, R)$. 
Proof. Let $\left\{\Gamma_{J K}^{I}\right\}$ be components of pseudo-Riemannian connection on TM. It follows from [8] that

$$
\begin{array}{llll}
\Gamma_{j k}^{i}=\Gamma_{j k}^{i}, & \Gamma_{j k}^{i}=0, & \Gamma_{j k}^{i}=0, & \Gamma_{j k}^{i}=0, \\
\Gamma_{j k}^{i}=\sum_{l=1}^{m} \frac{\partial \Gamma_{j k}^{i}}{\partial x^{l}} \xi^{l}, & \Gamma_{j k}^{i}=\Gamma_{j k}^{i}, & \Gamma_{j k}^{i}=\Gamma_{j k}^{i}, & \Gamma_{j k}^{i}=0 .
\end{array}
$$

If $Z(t)=(y(t), \eta(t))=\left\{y^{i}(t), \eta^{i}(t)\right\}$ is a parallel vector field along the curve $\alpha(t)=(c(t), \xi(t))=\left\{c^{i}(t), \xi^{i}(t)\right\}$ then $y^{i}(t)$ and $\eta^{i}(t)$ must satisfy the equations

$$
\frac{d y^{i}}{d t}+\sum_{j, k=1}^{m} \Gamma_{j k}^{i} \frac{d c^{j}}{d t} y^{k}=0
$$

(2) $\frac{d \eta^{i}}{d t}+\sum_{j, k=1}^{m}\left(\sum_{l=1}^{m} \frac{\partial \Gamma_{j k}^{i}}{\partial x^{l}} \xi^{l} \frac{d c^{j}}{d t} y^{k}+\Gamma_{j k}^{i} \frac{d c^{j}}{d t} \eta^{k}+\Gamma_{j k}^{i} \frac{d \xi^{j}}{d t} y^{k}\right)=0$.

If $A_{j}^{i}(t)$ denotes the components of the $m \times m$ matrix

$$
A(t)=\exp \int_{0}^{t}-\Gamma\left(\frac{d c}{d s}\right) d s,
$$

the solutions of the systems (1) and (2) are given by

$$
\begin{gathered}
y^{i}(t)=\sum_{j=1}^{m} A_{j}^{i}(t) y^{j}(0) \\
\eta^{i}(t)=\sum_{\lambda, \mu=1}^{m} A_{\lambda}^{i}(t) \int_{0}^{t}\left(A^{-1}\right)_{\mu}^{\lambda} \\
\quad \times\left\{\sum_{j, k=1}^{m}\left(-\sum_{l=1}^{m} \frac{\partial \Gamma_{j k}^{\mu}}{\partial x^{l}} \xi^{l} \frac{d c^{j}}{d s} y^{k}-\Gamma_{j k}^{\mu} \frac{d \xi^{j}}{d s} y^{k}\right)\right\} d s+\sum_{j=1}^{m} A_{j}^{i}(t) \eta^{j}(0) .
\end{gathered}
$$

Integrating by parts the second term on the right hand side of (4) and using equation (1), we get

$$
\begin{aligned}
\eta^{i}(t)= & -\left.\sum_{j, k=1}^{m} \Gamma_{j k}^{i}(c(t)) \xi^{j} y^{k}\right|_{0} ^{t}+\sum_{\lambda, \mu=1}^{m} A_{\lambda}^{i}(t) \int_{0}^{t}\left(A^{-1}\right)_{\mu}^{\lambda} \\
& \times \sum_{j, k, l=1}^{m} R_{k i j}^{\mu} \frac{d c^{j}}{d s} \xi^{l} y^{k} d s+\sum_{j=1}^{m} A_{j}^{i}(t) \eta^{j}(0) .
\end{aligned}
$$

Setting $t=1$ and writing equations (3) and (5) in vector form we get

$$
\begin{gathered}
y(1)=A(1) y(0) \\
\eta(1)=A(1) \int_{0}^{1} A(t)^{-1} R\left(\frac{d c}{d t}, \xi\right) A(t) y(0) d t+A(1) \eta(0)
\end{gathered}
$$


where $R$ is the curvature tensor of $M$. Here we have used the fact that $c(0)=c(1)=p, \Gamma_{j k}^{i}(p)=0$. Equations (6) and (7) imply that $A(1)=a \in$ $\Phi^{*}(p, M)$ and $\left(\begin{array}{cc}a & 0 \\ b & a\end{array}\right)=\tilde{a} \in \Phi^{*}(u, T M)$. Q.E.D.

3. Tangent bundle admits Sasaki metric. Suppose now that $\Phi^{*}(u, T M)$ denotes the holonomy group of the Sasaki connection on $T M$. For $p \in M$ and $u \in \tau^{-1}(p)$, let $U$ and $V$ be neighborhoods of $p$ in $M$ and of $u$ in $T M$ respectively such that (i) $U$ and $V$ are simply connected, (ii) $\tau(V)=U$, (iii) $\Phi^{*}(p, M)=\Phi^{0}(p, U)$, and (iv) $\Phi^{*}(u, T M)=\Phi^{0}(u, V)$. For any vector field $X$ on $M$, let $X^{h}$ and $X^{v}$ be the horizontal lift and vertical lift of $X$ respectively [1], [4]. If $\left\{Y^{1}, Y^{2}, \cdots, Y^{m}\right\}$ is an orthonormal base for vector fields on $U$, then $\left\{Y^{i h}, Y^{i v}\right\}_{i=1}^{m}$ forms a base for vector fields on $T M$, endowed with Sasaki metric. Let $\Gamma_{J K}^{I}$ be components of the Sasaki connection of $T M$ relative to the base $\left\{Y^{i h}, Y^{i v}\right\}_{i=1}^{m}$, and let $\Gamma_{j k}^{i}$ be components of the Riemannian connection of $T M$ relative to the base $\left\{Y^{i}\right\}_{i=1}^{m}$; from formulae on Sasaki connection [4, p. 125], we have the following

$$
\begin{array}{llll}
\Gamma_{j k}^{i}=\Gamma_{j k}^{i}, & \Gamma_{j k}^{i}=\frac{1}{2} \sum_{l=1}^{m} R_{k l j}^{i} \xi^{l}, & \Gamma_{j k}^{i}=\Gamma_{j k}^{i}, & \Gamma_{j k}^{i}=0, \\
\Gamma_{j k}^{i}=\frac{1}{2} \sum_{l=1}^{m} R_{l j k}^{i} \xi^{l}, & \Gamma_{j k}^{i}=0, & \Gamma_{j k}^{i}=\Gamma_{j k}^{i}, & \Gamma_{j k}^{i}=0 .
\end{array}
$$

We note that $\Gamma_{j k}^{i}=-\Gamma_{j i}^{k}$ and $\Gamma_{J K}^{I}=-\Gamma_{J I}^{K}$. Let $\alpha(t)=\left\{c^{i}(t), \xi^{i}(t)\right\}$ be a closed curve starting and ending at $u$ contained in $V$. If $\tilde{a}$ is an element in $\Psi^{*}(u, T M)$ defined by $\alpha(t)$, it follows from the lemma in $\$ 2$ that $\tilde{a}=\exp \int_{0}^{1} \Gamma(d \alpha / d t) d t$ where the $2 m \times 2 m$ matrix $\Gamma(d \alpha / d t)$ is given as follows:

$$
\Gamma\left(\frac{d \alpha}{d t}\right)=\left(\begin{array}{cc}
\Gamma\left(\frac{d c}{d t}\right)+\frac{1}{2} R\left(\xi, \frac{d \xi}{d t}\right) & \frac{1}{2} R(\xi,) \frac{d c}{d t} \\
-\frac{1}{2} R\left(\frac{d c}{d t},\right) \xi & \Gamma\left(\frac{d c}{d t}\right)
\end{array}\right)
$$

We note that $\Gamma(d \alpha / d t)$ is skew-symmetric. If $\xi(t)=0$ in the local expression $\alpha(t)=(c(t), \xi(t))$, the element $\tilde{a} \in \Phi^{*}(u, T M)$ defined by $\alpha(t)=(c(t), 0)$ is given by

$$
\tilde{a}=\int_{0}^{1} \Gamma\left(\frac{d \alpha}{d t}\right) d t=\left(\begin{array}{cc}
\exp \int_{0}^{1} \Gamma(d c / d t) d t & 0 \\
0 & \exp \int_{0}^{1} \Gamma(d c / d t) d t
\end{array}\right) .
$$

THEOREM 2. Every element of the local holonomy group $\Phi^{*}(u, T M)$ can be expressed in the form $\exp \int_{0}^{1} \Gamma(d \alpha / d t) d t$ where $\Gamma(d \alpha / d t)$ is a skelisymmetric $2 m \times 2 m$ matrix. $\left\{\left(\begin{array}{cc}a & 0 \\ 0 & a\end{array}\right) \mid a \in \Phi^{*}(p, m)\right\}$ is a subgroup of $\Phi *(u, T M)$. 
4. Parallel sections. Suppose that $M$ is a connected manifold, and that $\Pi: E \rightarrow M$ is a vector bundle over $M$ associated with a principal fibre bundle $P \rightarrow M$. Let $\nabla$ be a connection on $P$. The holonomy group of $P$ is also called the holonomy group of $E$, denoted by $\Phi(\Pi)$. A section $\varphi$ of $E$ is called parallel if $\nabla \varphi=0$. $\varphi$ is a parallel section of $E$ if and only if $\varphi(p)$ is left fixed by $\Phi(\Pi)$ for a point $p$ in $M$. Let $E_{0}$ be a subspace of $\Pi^{-1}(p)$ on which $\Phi(\Pi)$ acts trivially, and let $E_{1}$ be a subspace of parallel sections of $E$. Then we have $\operatorname{dim} E_{0}=\operatorname{dim} E_{1}$.

THEOREM 3. If $\Pi: E \rightarrow M$ is an orthogonal vector bundle of $\operatorname{dim} 2$, then $E$ is locally flat if and only if there exists a parallel section of $E$.

ProOF. Since the restricted holonomy group $\Phi^{0}(\Pi)$ is a subgroup of $O(2)$, the orthogonal group of degree $2, \Phi^{0}(\Pi)$ is a discrete group consisting of identity alone if and only if $E_{0} \neq 0$. From Theorem 4.1 [3, p. 210], $E$ is locally flat if and only if $\Phi^{0}(\Pi)$ is discrete. The theorem follows from the above two facts. Q.E.D.

Suppose $\Pi: E \rightarrow M$ and $\rho: F \rightarrow M$ are vector bundles over $M$. Let $R_{\Pi}$ and $R_{\rho}$ be curvature tensors for the bundles $\Pi$ and $\rho$ respectively. Let $L(\Pi, \rho): L(E, F) \rightarrow M$ be the vector bundle of linear maps of the fibers, and let $R_{L(\Pi, \rho)}$ be a curvature tensor for $L(\Pi, \rho)$. A vector bundle $E$ is said to be locally symmetric if $\nabla R_{\mathrm{II}}=0$. By a long direct calculation, we have $\nabla_{w} R_{L(\Pi, \rho)}(v, u, A) \cdot \xi=\left(\nabla_{w} R_{\rho}\right) \cdot(v, u, A \cdot \xi)-A\left(\nabla_{w o} R_{\Pi}\right) \cdot(v, u, \xi)$ for $u, v, w \in T_{p} M, A \in L\left(E_{p}, F_{p}\right)$ and $\xi \in E_{p}$. It implies the following

Proposition. If $\Pi: E \rightarrow M$ and $\rho: F \rightarrow M$ are locally symmetric vector bundles, then $L(\Pi, \rho): L(E, F) \rightarrow M$ is also locally symmetric.

If we set $\Pi^{*}: E^{*}=L(E, R) \rightarrow M$, where $R$ is the set of real numbers, then $E^{*}$ is the dual bundle of $E$ and has an induced connection from $E$. Since $L(E, F) \cong E^{*} \otimes F, E^{*} \otimes F$ has an induced connection from $L(E, F)$. For simplicity, let $\nabla$ denote the connections of $E, F$ and also the induced connections. Let $\Im(E)$ denote the space of differentiable sections of $E$. If $e^{*} \in \mathcal{S}(E)$ and $f \in \mathcal{S}(F)$, it is easy to see that $e^{*} \otimes f \in \mathcal{S}\left(E^{*} \otimes F\right)$ and $\nabla\left(e^{*} \otimes f\right)=\nabla e^{*} \otimes f+e^{*} \otimes \nabla f$.

TheOREM 4. Suppose $\Pi: E \rightarrow M$ and $\rho: F \rightarrow M$ are vector bundles over $M$ with connections. Then the holonomy group $\Phi(L(\Pi, \rho))$ is a subgroup of $\Phi(\Pi) \otimes \Phi(\rho)$.

Proof. First we show that $\Phi\left(\Pi^{*}\right) \cong \Phi(\Pi)$. Suppose that $c(t)$ is a closed curve in $M$ starting and ending at $p$, and that $\left\{e_{i}\right\}$ is a base of $E_{p}$. Let $e_{i}(t)$ be the parallel section along $c(t)$ with $e_{i}(0)=e_{i}$, and let $e_{i}^{*}(t)$ be a section in $E^{*}$ dual to $e_{i}(t)$, that is, $e_{i}^{*}(t) \cdot e_{j}(t)=\delta_{i j}$ for $0 \leqq t \leqq 1$. 
We assert that $e_{i}^{*}(t)$ is a parallel section of $E^{*}$ with $e_{i}^{*}(0)=e_{i}^{*}$. In fact, since $0=\nabla\left(e_{i}^{*} \cdot e_{j}\right)=\nabla e_{i}^{*} \cdot e_{j}+e_{i}^{*} \cdot \nabla e_{j}$ and $\nabla e_{j}=0$, it follows that $\nabla e_{i}^{*}$. $e_{j}=0$ for all $i ; j$, and hence $\nabla e_{i}^{*}=0$. If $\alpha(t)$ is a parallel section along $c(t)$ in $E$ with $\alpha(0)=\alpha_{0}$, then $\alpha^{*}(t)$ is a parallel section along $c(t)$ in $E^{*}$ with $\alpha^{*}(0)=\alpha_{0}^{*}$. It implies that $\Phi\left(\Pi^{*}\right) \cong \Phi(\Pi)$. Let $\left\{f_{j}\right\}$ be a base of $F_{p}$, and let $f_{j}(t)$ be a parallel section along $c(t)$ in $F$ with $f_{j}(0)=f_{j}$. We identify $\Phi\left(\Pi^{*}\right)$ with $\Phi(\Pi)$, and $L(E, F)$ with $E^{*} \otimes F$. Now every element $A \in$ $L\left(E_{p}, F_{p}\right)$ can be expressed as $A=\sum_{i, j} d_{i j} e_{i}^{*} \otimes f_{j}$. It follows that $A(t)=$ $\sum_{i, j} d_{i j} e_{i}^{*}(t) \otimes f_{j}(t)$ is a parallel section of $L(E, F)$ along $c(t)$ with $A(0)=A$. If the closed curve $c(t)$ defines an element $a$ in $\Phi(\Pi)$ and $b$ in $\Phi(\rho)$ respectively, then $A(1)=(a \otimes b) A$. This proves that $\Phi(L(\Pi, \rho))$ is a subgroup of $\Phi(\Pi) \otimes \Phi(\rho)$.

Let $\tau: T M \rightarrow M$ be the tangent bundle of $M$ with a connection. An iterated bundle $L^{i+1}(T M, E)$ is defined by

$$
L^{i+1}(T M, E)=L\left(T M, L^{i}(T M, E)\right) .
$$

COROLlaRY 4.1. The holonomy group of $L^{i}(T M, E)$ is a subgroup of $\otimes^{i} \Phi(\tau) \otimes \Phi(\Pi)$.

Let $S_{\mathrm{\Pi}}$ denote the subspace of the parallel sections of $\Pi: E \rightarrow M$. By using the fact that $\varphi \in S_{\Pi}$ if and only if $\Phi(\Pi)$ leaves $\varphi(p)$ fixed for a point $p$ in $M$, we have

Corollary 4.2. $\operatorname{dim} S_{L(\Pi, \rho)} \geqq \operatorname{dim} S_{\Pi} \times \operatorname{dim} S_{\rho}$.

We note that a fiber map $f: E \rightarrow F$ is affine if and only if $f$ is a parallel section of $L(E, F)$.

THEOREM 5. Suppose $M$ is a connected, simply connected Riemannian manifold. If the euclidean space $M_{0}$ in the de Rham decomposition of $M$ is nontrivial, then there exist affine fiber maps from TM into TM.

Proof. We first note that $\operatorname{dim} M_{0}=\operatorname{dim} S_{r} \neq 0$. From Corollary 4.2 , it follows that $\operatorname{dim} S_{L(r, r)} \neq 0$, and hence there exist affine fiber maps. Q.E.D.

COROLlARY 5.1. Suppose $M$ is a connected, simply connected Riemannian manifold with vanishing Ricci curvature. Then there. exists an infinitesimal isometry on $M$ if and only if the euclidean space $M_{0}$ in the de Rham decomposition is nontrivial.

Proof. From Proposition 2.5 [3, p. 235] and the argument used in the proof of Corollary $5.4[3$, p. 251$]$, it follows that $X$ is an infinitesimal isometry if and only if $X$ is a parallel vector field. The corollary then follows from the fact that $\operatorname{dim} M_{0}=\operatorname{dim} S_{r}$. Q.E.D. 
THEOREM 6. Suppose that $M$ and $N$ are affine manifolds, and that $f: M \rightarrow N$ is a differentiable map. If there exist parallel vector fields on $M$ and $N$ respectively. Then there exist affine fiber maps $F: T M \rightarrow T N$ such that $f \circ \tau_{M}=\tau_{N} \circ F$.

Proof. It suffices to prove that $\operatorname{dim} S_{L\left(r_{M}, f^{*} \tau_{N}\right)} \neq 0$. Let $\nabla$ and $\nabla^{\prime}$ denote affine connections on $M$ and $N$ respectively. The pullback bundle $f^{*} \tau_{N}: f^{*} T N \rightarrow M$ has a naturally induced connection $f^{*} \nabla^{\prime}$. A vector field $X$ on $N$ induces a section $f^{*} X=X \circ f$ on $f^{*} \tau_{N}$. It follows from [2] that $\left(f^{*} \nabla^{\prime}\right)\left(f^{*} X\right)=f^{*}\left(\nabla^{\prime} X\right) \circ f_{*}$, where $f_{*}: T M \rightarrow T N$ is the tangent map of $f$. So $f^{*} X$ is a parallel section of $f^{*} \tau_{N}$ if $X$ is a parallel vector field of $N$, which implies $\operatorname{dim} S_{f{ }^{*} \tau_{N}}>0$. From Corollary 4.2 and assumptions, we get $\operatorname{dim} S_{L\left(\tau_{M}, *^{*} \tau_{N}\right)}>0$. Q.E.D.

\section{REFERENCES}

1. P. Dombrowski, On the geometry of the tangent bundle, J. Reine Angew Math. 210 (1962), 73-88. MR 25 \#4463.

2. H. Eliasson, Geometry of manifolds of maps, J. Differential Geometry 1 (1967), 169194. MR 37 \#2268.

3. S. Kobayashi and K. Nomizu, Foundations of differential geometry. Vol. I, Interscience, New York, 1963. MR 27 \#2945.

4. O. Kowalski, Curvature of the induced Riemannian metric on the tangent bundle of a Riemannian manifold, J. Reine Angew Math. 250 (1971), 124-129. MR 44 \#3244.

5. S. Sasaki, On the differential geometry of tangent bundles of Riemannian manifolds. I, II, Tôhoku Math. J. (2) 10 (1958), 338-354; ibid. (2) 14 (1962), 146-155. MR 22 \#3007; 26 \#2987.

6. J. Vilms, Connections on tangent bundles, J. Differential Geometry 1 (1967), 235-243. MR 37 \#742.

7. — Totally geodesic maps, J. Differential Geometry 4 (1970), 73-79. MR 41 \#7589.

8. (a) K. Yano and S. Kobayashi, Prolongations of tensor fields and connections to tangent bundles. I. General theory, J. Math. Soc. Japan 18 (1966), 194-210. MR 33 \#1814.

(b) Prolongations of tensor fields and connections to tangent bundles. II. Infinitesimal automorphisms, J. Math. Soc. Japan 18 (1966), 236-246. MR 34 \#743.

(c) Prolongations of tensor fields and connections to tangent bundles. III. Holonomy groups, J. Math. Soc. Japan 19 (1967), 486-488. MR 36 \#2084.

Department of Mathematics, University of Illinois, Chicago, Illinois 60680 